\title{
The Mimetic Finite Difference Discretization of Diffusion Problem on Unstructured Polyhedral Meshes
}

\author{
Konstantin Lipnikov* Mikhail Shashkov* Daniil Svyatskiy ${ }^{\dagger \ddagger}$
}

\begin{abstract}
We study the mimetic finite difference discretization of diffusion-type problems on unstructured polyhedral meshes. We demonstrate high accuracy of the approximate solutions for general diffusion tensors, the second-order convergence rate for the scalar unknown and the first order convergence rate for the vector unknown on smooth or slightly distorted meshes, on non-matching meshes, and even on meshes with irregular-shaped polyhedra with flat faces. We show that in general the meshes with non-flat faces require more than one flux unknown per mesh face to get optimal convergence rates.
\end{abstract}

\section{Introduction}

The development of new mathematical models and numerical methods results in the need for discretization methods handling unstructured polyhedral meshes. For instance, such meshes appear in the basin modeling where mesh cells have to approximate sophisticated geological structures. Other examples come from applications using adaptive mesh refinement algorithms or non-matching meshes where some of the mesh elements are degenerate and even non-convex polyhedra.

The mimetic finite difference discretization has been successfully employed for solving problems of continuum mechanics [14], electromagnetics [8], gas dynamics [5], and linear diffusion on simplicial and polygonal meshes in both the Cartesian and polar coordinates $[7,9,10,13,16]$. The family of mimetic discretizations contains the classical mixed finite element discretizations (on tetrahedral and hexahedral meshes) [17] and the symmetric version [3] of multi-point flux approximation methods [1].

The discretization methodology is based on the support operator approach (see [7] and references therein). According to this approach, the constructed discrete operators have to preserve critical properties of the original continuous differential operators.

The work was performed at Los Alamos National Laboratory operated by the University of California for the US Department of Energy under contract W-7405-ENG-36.

${ }^{*}$ Los Alamos National Laboratory, MS B284, Los Alamos, NM, 87545, lipnikov, shashkov@lanl.gov

${ }^{\dagger}$ University of Houston, Department of Mathematics, Houston, 77204, dasvyat@math.uh.edu

† The corresponding author. 
Conservation law, solution symmetries and relationships between differential operators are examples of such properties. For the linear diffusion problem, this means that the mimetic discretization mimics (a) the Gauss divergence theorem to enforce the local conservation law, (b) the symmetry between the gradient and divergence operators, $\mathcal{G}=\mathcal{D} I V^{*}$, to guarantee symmetry and positivity of the discrete operator $\mathcal{D} I V \mathcal{G}$, and (c) the null spaces of the involved operators to prove stability of the discretization.

In this paper, we consider a general diffusion problem on a conformal polyhedral partition. The diffusion problem is formulated as a first-order system consisting of the mass balance equation and the constitutive equation describing the relationship between the vector unknown (flux) and the scalar unknown (pressure). The later equation is known as the Darcy law in porous media applications. We assume that the material properties are described by a $3 \times 3$ full tensor.

The discretization methodology employes the divide and conquer principle. First, we consider each mesh polyhedron as a separate domain and construct an independent discretization for this polyhedron. If the polyhedron has flat faces and the diffusion tensor is constant, this discretization will be exact for linear pressures. Second, the system of element-based discretizations is closed by imposing continuity and boundary conditions on polyhedron faces for pressure unknowns and normal flux components.

For sufficiently smooth solutions, it was proved in [4] that the mimetic discretization is second order accurate for the pressure unknown on unstructured polyhedral meshes having degenerate and non-convex polyhedra with flat faces. In this paper, we extend the analysis to meshes with more general polyhedra. If the faces of a polyhedron are not flat, we shall refer to them as curved faces. We show with numerical experiments that the optimal convergence rates are held for polyhedral meshes with slightly curved faces. More specifically, the deviation from a flat face should be of order $h^{2}$ where $h$ is the local mesh size. In the case of meshes with strongly curved faces (see Section 6.3), we show that more than one flux unknown per curved face is required to get the optimal convergence rate. To the best of our knowledge, there is no locally conservative discretization method which uses 1 flux unknown per mesh face, 1 pressure unknown per mesh element and converges on randomly perturbed meshes.

More than one flux unknown per mesh face is also used in the multi-point flux approximation method (see e.g. $[1,15]$ ). However, the method results in the discretization scheme with a non-symmetric coefficient matrix whose positivity has not been proved yet. The recently proposed mimetic finite element method $[11,12]$ on polyhedral meshes is close to our method, but its convergence on randomly perturbed meshes has not been studied yet. The advantage of our approach is flexibility imbedded in definition of inner products.

The results obtained in this paper are fundamental extensions of the $2 \mathrm{D}$ results presented in [10]. In particular, we address new issues related to curved mesh faces and propose a new algorithm for computing interior fluxes through boundary fluxes.

The paper is organized as follows. In Section 2, we formulate the general diffusion problem. In Section 3.1, we describe the local support operator approach and build the mimetic discretization over a single mesh polyhedron. In Section 3.2, we impose interface conditions and determine how the boundary conditions are involved in the discretization. In Section 4, we derive the inner product in the space of fluxes. The solution algorithm 
is described in Section 5. The accuracy and robustness of our discretization method are analyzed numerically in Section 6.

\section{Problem formulation}

Let us consider the 3D linear diffusion equation

$$
-\operatorname{div}(K \operatorname{grad} p)+c p=Q \quad \text { in } \quad \Omega,
$$

where $p$ is an unknown scalar function that we refer to as the pressure, $K=K(\mathbf{x})$ is a full diffusion tensor, $c(\mathbf{x})$ is a nonnegative function, $Q(\mathbf{x})$ is a source function, and $\Omega \subset \mathbb{R}^{3}$ is a bounded domain. We assume that $K$ is a symmetric, bounded uniformly positive definite matrix, i.e.

$$
\alpha_{1}(\boldsymbol{\xi}, \boldsymbol{\xi}) \leq(K(\mathbf{x}) \boldsymbol{\xi}, \boldsymbol{\xi}) \leq \alpha_{2}(\boldsymbol{\xi}, \boldsymbol{\xi}), \quad \forall \boldsymbol{\xi} \in \mathbb{R}^{3}, \quad \forall \mathbf{x} \in \bar{\Omega}
$$

with a positive constants $\alpha_{1}$ and $\alpha_{2}$ independent of $\mathbf{x}$ and $\boldsymbol{\xi}$. We assume that the domain boundary $\partial \Omega$ is partitioned into two non overlapping sets $\Gamma_{D}$ and $\Gamma_{R}$ such that $\Gamma_{D}$ is a closed set and the closure of $\Gamma_{D} \cup \Gamma_{R}$ is equal to $\partial \Omega$.

Equation (2.1) is complemented with two boundary conditions

$$
\begin{aligned}
p & =g_{D} \text { on } \Gamma_{D}, \\
(K \operatorname{grad} p) \cdot \mathbf{n}+\sigma p & =g_{R} \text { on } \Gamma_{R},
\end{aligned}
$$

where $\mathbf{n}$ is the outward unit normal vector to $\Gamma_{R}, \sigma(\mathbf{x})$ is a nonnegative function, and $g_{D}$ and $g_{R}$ are given functions. We also assume that the data are smooth enough so that problem (2.1)-(2.2) has a unique solution [6].

We replace the second order problem $(2.1)$ by an equivalent system of first-order equations:

$$
\begin{aligned}
\mathbf{F}+K \operatorname{grad} p & =0 \text { in } \Omega, \\
\operatorname{div} \mathbf{F}+\quad c p & =Q \text { in } \Omega,
\end{aligned}
$$

where $\mathbf{F}$ denotes a vector-valued function that we refer as the flux.

Let $\Omega_{h}$ be a non-overlapping conformal partition of $\Omega$ into polyhedral elements $e_{i}$ :

$$
\Omega_{h}=\bigcup_{i=1}^{N} e_{i} .
$$

The element $e_{i}$ is a closed simply-connected "generalized" polyhedron. In particular, $e_{i}$ may be a non-convex polyhedron or may have $2 \mathrm{D}$ angles equal to $\pi$. However, we assume that there are no zero 2D angles. The conformal partition implies that closures of any two elements have either a common vertex, or a common whole edge, or a common whole face, or do not intersect.

A curved (non-planar) face of a "generalized" polyhedron is defined by a set of ordered vertices in $3 \mathrm{D}$ which makes its precise definition a non-trivial task. We assume that there exists a one-to-one map which transforms the curved face to a regular (flat) 
polygon. Many production codes using polyhedral meshes specify this map by approximating the curved face with a piecewise linear surface. In this paper, we employ the same approach. First, we define an arbitrary point and call it the center of the curved face. Second, we connect this point with vertices of the curved face to get a triangular piecewise linear surface which we still refer to as the curved face.

It is obvious to require that the curved faces defined by the piecewise linear surfaces do not overlap and result in the valid mesh. In numerical experiments, we use the geometric center as the face center and perform a few checks of mesh validity.

Later, we use notation of a polyhedron center. In general, the center of polyhedron $e$ can be an arbitrary point inside $e$. However, to simplify the presentation, we assume that this point is the center of mass of $e$. We shall also refer to a "generalized" polyhedron simply as a polyhedron.

\section{Mimetic finite difference method}

Let us integrate the mass balance equation (the second equation in (2.3)) over arbitrary polyhedron $e$. The Gauss divergence theorem gives

$$
\sum_{A \in \partial E} \int_{A} \mathbf{F} \cdot \mathbf{n} \mathrm{d} A+\int_{e} c p \mathrm{~d} V=\int_{e} Q \mathrm{~d} V .
$$

It implies that one of the natural choices for the discrete unknowns are the normal components of the flux averaged over the polyhedron faces and the pressure averaged over the polyhedron. The mimetic finite difference method uses these unknowns to discretize the constitutive equation (the first equation in (2.3)) and to preserve the essential properties of system (2.3). In order to formulate these properties, we introduce the generalized gradient, $\mathcal{G}$, and the divergence, $\mathcal{D}$, operators:

$$
\mathcal{G} p=-K \operatorname{grad} p \quad \text { and } \quad \mathcal{D} \mathbf{F}=\left\{\begin{array}{ccc}
\operatorname{div} \mathbf{F} & \text { on } e, \\
-\mathbf{F} \cdot \mathbf{n} & \text { on } \partial e .
\end{array}\right.
$$

For simplicity, we shall refer to the generalized gradient operator as the flux operator. Let us define the following inner products:

$$
(\mathbf{F}, \mathbf{H})_{\mathbb{X}}=\int_{e} \mathbf{F} \cdot K^{-1} \mathbf{H} \mathrm{d} V \quad \text { and } \quad(p, q)_{\mathbb{Q}}=\int_{e} p q \mathrm{~d} V+\int_{\partial e} p q \mathrm{~d} A .
$$

Then, the Gauss-Green theorem,

$$
\int_{e} \mathbf{F} \cdot \operatorname{grad} p \mathrm{~d} V+\int_{e} p \operatorname{div} \mathbf{F} \mathrm{d} V=\int_{\partial e} p \mathbf{F} \cdot \mathbf{n} \mathrm{d} A,
$$

gives us the following relationship between the generalized gradient and divergence operators:

$$
(\mathbf{F}, \mathcal{G} p)_{\mathbb{X}}=(\mathcal{D} \mathbf{F}, p)_{\mathbb{Q}}
$$

The last expression clearly states that these operators are adjoint to each other:

$$
\mathcal{G}=\mathcal{D}^{*}
$$

We show in the next section that the mimetic discretization preserves this property. 


\subsection{Discretization on element}

In this section, we describe the mimetic discretization over the polyhedron $e$. First, we introduce vector spaces of discrete functions and inner products on them. Then, we construct the discrete generalized divergence operator and derive the discrete flux operator as the adjoint to it.

We begin by specifying the degrees of freedom for physical variables $p$ and $\mathbf{F}$ and their location. The scalar unknowns are defined at the polyhedron center, $p^{0}$, and at the centers of its faces, $p^{1}, p^{2}, \ldots, p^{s}$, where $s$ is the number of polyhedron faces. We denote the vector space of discrete pressure functions $\vec{p}=\left(p^{0}, p^{1}, \ldots, p^{s}\right)^{T}$ as $\mathbb{Q}_{h}$. The dimension of $\mathbb{Q}_{h}$ is equal to $s+1$. The inner product on this space is given by

$$
(\vec{p}, \vec{q})_{\mathbb{Q}_{h}}=p^{0}\left|V_{e}\right|+\sum_{k=1}^{s} p^{k} q^{k}\left|A^{k}\right|, \quad \forall \vec{p}, \vec{q} \in \mathbb{Q}_{h},
$$

where $\left|V_{e}\right|$ is the volume of $e$ and $\left|A^{k}\right|$ is the area of the $k$-th face. It is not difficult to determine the relationship between this inner product and the standard dot product $<\cdot, \cdot>$ on the Euclidean space $\mathbb{R}^{s+1}$ :

$$
(\vec{p}, \vec{q})_{\mathbb{Q}_{h}}=<L \vec{p}, \vec{q}>, \quad L=\operatorname{diag}\left\{\left|V_{e}\right|,\left|A^{1}\right|, \ldots,\left|A^{s}\right|\right\} .
$$

The vector unknowns are defined as the face-normal components, $f^{1}, f^{2}, \ldots, f^{s}$, located at centers of polyhedron faces (see Fig. 1). For instance, $f^{1}$ approximates the dot product of $\mathbf{F}$ with the outward unit normal $\mathbf{n}^{1}$. Let $\vec{f}=\left(f^{1}, f^{2} \ldots f^{s}\right)^{T}$ be a vector of these face-normal components. We denote a discrete space containing such vectors as $\mathbb{X}_{h}$. The dimension of this space is equal to $s$. The inner product on $\mathbb{X}_{h}$ is defined as follows:

$$
(\vec{f}, \vec{g})_{\mathbb{X}_{h}}=<M \vec{f}, \vec{g}>, \quad \forall \vec{f}, \vec{g} \in \mathbb{X}_{h},
$$

where $M \in \mathbb{R}^{s \times s}$ is a symmetric positive-definite matrix. The choice of the matrix $M$ is crucial and one of the most difficult problems in the support operator approach. In particular, this inner product has to approximate the continuous one with sufficient accuracy. In the next section, we present an algorithm for constructing a suitable matrix $M$.

Now, we specify the discrete extended divergence operator. Based on the Gauss divergence theorem,

$$
\int_{e} \operatorname{div} \mathbf{F} \mathrm{d} V=\int_{\partial e} \mathbf{F} \cdot \mathbf{n} \mathrm{d} A,
$$

and definition of discrete unknowns, we define the discrete divergence operator,

$$
\mathcal{D} I V^{h} \vec{f} \equiv \frac{1}{\left|V_{e}\right|} \sum_{k=1}^{s} f^{k}\left|A^{k}\right|,
$$

and the generalized discrete divergence operator:

$$
\mathcal{D}^{h} \vec{f} \equiv\left(\mathcal{D} I V^{h} \vec{f},-f^{1},-f^{2}, \ldots,-f^{s}\right)^{T} .
$$



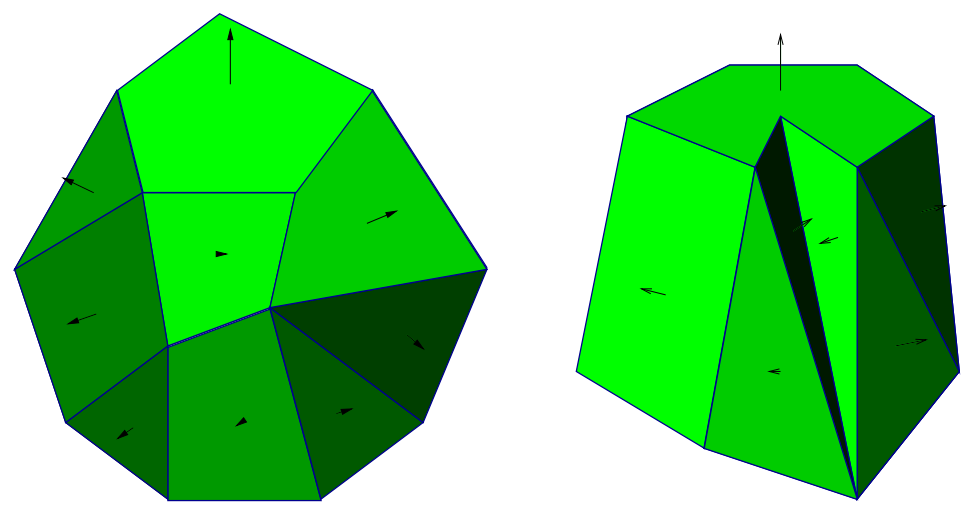

Figure 1: Two possible elements and the normals to their faces.

Finally we derive the discrete flux operator $\mathcal{G}^{h}$ which is adjoint to $\mathcal{D}^{h}$ with respect to the inner products defined on spaces $\mathbb{X}_{h}$ and $\mathbb{Q}_{h}$ :

$$
\left(\vec{f}, \mathcal{G}^{h} \vec{p}\right)_{\mathbb{X}_{h}}=\left(\vec{p}, \mathcal{D}^{h} \vec{f}\right)_{\mathbb{Q}_{h}}, \quad \forall \vec{p} \in \mathbb{Q}_{h}, \quad \forall \vec{f} \in \mathbb{X}_{h}
$$

Since vector $\vec{f}$ is arbitrary, formula (3.4) implies that

$$
\mathcal{G}^{h} \vec{p}=M^{-1} \hat{L}\left(\begin{array}{c}
p^{0}-p^{1} \\
\vdots \\
p^{0}-p^{s}
\end{array}\right), \quad \hat{L}=\operatorname{diag}\left\{\left|A^{1}\right|, \ldots,\left|A^{s}\right|\right\} .
$$

It is pertinent to note that one of the important properties of the continuous flux operator is that $\operatorname{grad} p=0$ if and only if $p$ is a constant function. It is highly desirable to have the same property for the null space of the discrete flux operator. Indeed, if a high-frequency mode enters the null space of the discrete gradient operator, a special procedure for filtering noise from the solution will be required. Since $M$ is the positive definite matrix, the null space of $\mathcal{G}$ contains only constant vectors.

The definitions of the discrete divergence and gradient operators result in the following local equations:

$$
\begin{aligned}
\vec{f} & =\mathcal{G}^{h} \vec{p}, \\
\mathcal{D} I V^{h} \vec{f}+c_{e} p^{0} & =Q_{e},
\end{aligned}
$$

where

$$
Q_{e}=\frac{1}{\left|V_{e}\right|} \int_{e} Q(\mathbf{x}) \mathrm{d} V \quad \text { and } \quad c_{e}=\frac{1}{\left|V_{e}\right|} \int_{e} c(\mathbf{x}) \mathrm{d} V
$$

\subsection{Interface conditions}

The system of discrete equations (3.6) is closed by imposing continuity conditions on mesh faces for primary variables $p$ and $\mathbf{F}$. Hereafter, we shall use the subscript $i$ for vectors, matrices and inner products which are associated with the polyhedron $e_{i}$ and the superscript $k$ for polyhedron faces. For instance, $\left|A_{i}^{k}\right|$ is the area of the $k$-th faces of $e_{i}$. The corresponding pressure variable located on that face is $p_{i}^{k}$ and the normal 
component of the flux is $f_{i}^{k}$. For the continuous problem (2.3), we have continuity of the pressure and of the normal component of the flux across mesh faces. For the discrete problem, it means that:

$$
f_{i_{1}}^{k_{1}}=-f_{i_{2}}^{k_{2}} \quad \text { and } \quad p_{i_{1}}^{k_{1}}=p_{i_{2}}^{k_{2}}
$$

if polyhedron $e_{i_{1}}$ shares its $k_{1}$-th face with the $k_{2}$-th face of polyhedron $e_{i_{2}}$.

Furthermore, we determine how the boundary conditions are involved in the mimetic discretization. If the $k$-th face of the polyhedron $e_{i}$ belongs to $\Gamma_{D}$, then the corresponding pressure unknown, $p_{i}^{k}$, is equal to:

$$
p_{i}^{k}=\frac{1}{\left|A_{i}^{k}\right|} \int_{A_{i}^{k}} g_{D}(\mathbf{x}) \mathrm{d} A .
$$

If this face belongs to $\Gamma_{N}$, then $p_{i}^{k}$ and $f_{i}^{k}$ satisfy to the following relations:

$$
-f_{i}^{k}+\sigma_{i}^{k} p_{i}^{k}=\frac{1}{\left|A_{i}^{k}\right|} \int_{A_{i}^{k}} g_{R}(\mathbf{x}) \mathrm{d} A \quad \text { where } \quad \sigma_{i}^{k}=\frac{1}{\left|A_{i}^{k}\right|} \int_{A_{i}^{k}} \sigma(\mathbf{x}) \mathrm{d} A .
$$

\section{Scalar product in the discrete flux space}

In this section we derive the inner product in the space of fluxes for a polyhedron $e$. The derivation is based on a partition of $e$ into tetrahedra. Note that this partition is obviously not unique.

We described in Section 2, how to split the curved face into triangles. Now, connecting the polyhedron center with vertices of these triangles, we get one possible decomposition of $e$ into tetrahedra. An example of such a partition is shown in Fig. 2.

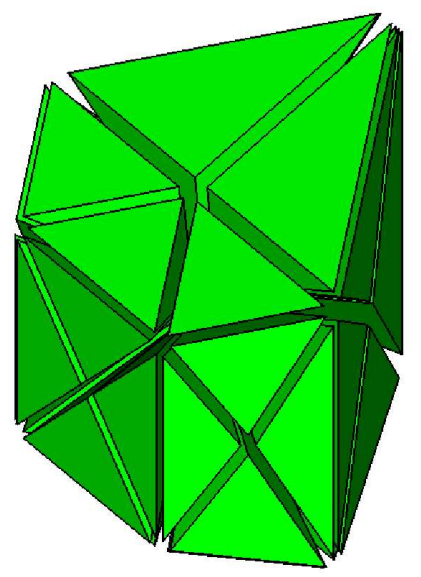

Figure 2: The partition of a polyhedron into tetrahedra.

To construct an inner product over a polyhedron, we use an accurate inner product over a tetrahedron [7]. We assume the medium in each tetrahedron is homogeneous but material properties (diffusion tensor) may vary between tetrahedra. 


\subsection{Derivation of the inner product over a tetrahedron}

Consider a single tetrahedron $\Delta$. Note, that the whole flux (a 3D vector) can be recovered at each vertex of $\Delta$ from three normal components associated with three adjacent triangles. We denote the recovered vectors by $\mathbf{F}_{\mathbf{k}}$ where $k$ is the vertex number, $k=1, \ldots, 4$. Suppose that the $k$-th vertex belongs to the faces with indices $i_{1}, i_{2}, i_{3}$. Then, the vector $\mathbf{F}_{\mathbf{k}}$ can be recovered using the corresponding face-normal components $f^{i_{1}}, f^{i_{2}}$ and $f^{i_{3}}$. Let $\mathbf{n}^{\mathbf{i}_{\mathbf{k}}}=\left(n_{x}^{i_{k}}, n_{y}^{i_{k}}, n_{z}^{i_{k}}\right)$ be the unit outer normal to the $i_{k}$-th face. Then,

$$
\mathbf{F}_{\mathbf{k}}=\left(\begin{array}{ccc}
n_{x}^{i_{1}} & n_{y}^{i_{1}} & n_{z}^{i_{1}} \\
n_{x}^{i_{2}} & n_{y}^{i_{2}} & n_{z}^{i_{2}} \\
n_{x}^{i_{3}} & n_{y}^{i_{3}} & n_{z}^{i_{3}}
\end{array}\right)^{-1}\left(\begin{array}{l}
f^{i_{1}} \\
f^{i_{2}} \\
f^{i_{3}}
\end{array}\right)
$$

With the recovered vectors, the continuous inner product can be approximated as follows:

$$
\int_{\Delta} K^{-1} \mathbf{F} \cdot \mathbf{H} \mathrm{d} V \approx \frac{\left|V_{\Delta}\right|}{4} \sum_{k=1}^{4} K_{\Delta}^{-1} \mathbf{F}_{\mathbf{k}} \cdot \mathbf{H}_{\mathbf{k}},
$$

where $\left|V_{\Delta}\right|$ is the volume of the tetrahedron $\Delta$ and $K_{\Delta}$ is the value of the diffusion tensor at the center of mass of $\Delta$. This approximation is obviously exact for constant fluxes and constant tensors. The right hand side of formula (4.1) can be written as follows:

$$
\frac{\left|V_{\Delta}\right|}{4} \sum_{k=1}^{4} K_{\Delta}^{-1} \mathbf{F}_{\mathbf{k}} \cdot \mathbf{H}_{\mathbf{k}}=<M_{\Delta} \vec{f}, \vec{h}>,
$$

where $\vec{f}=\left(f^{1}, f^{2}, f^{3}, f^{4}\right)^{T}, \vec{h}=\left(h^{1}, h^{2}, h^{3}, h^{4}\right)^{T}, M_{\Delta}$ is a $4 \times 4$ symmetric positive definite matrix and $\langle\cdot, \cdot\rangle$ is the standard dot product on the Euclidean space $\mathbb{R}^{4}$.

\subsection{Derivation of the inner product over a polyhedron}

According to the procedure described above the polyhedron $e$ can be partitioned into $t$ non-overlapping tetrahedra, $\Delta_{l}, l=1 \ldots t$. On each triangular face of this partition, we define a unit normal vector in such a way that on the face belonging to $\partial e$ the normal vector is outer. Moreover, we temporary introduce additional unknowns, normal components of the flux associated with these normal vectors. Let $\vec{f}{ }^{i n t}$ be the vector of auxiliary unknowns located on the internal faces (with respect to the polyhedron boundary) and $\vec{f}^{e x t}$ be the vector of auxiliary unknowns located on the remaining triangular faces. The dimension of vectors $\vec{f}^{i n t}$ and $\vec{f}^{e x t}$ is denoted by $n_{i}$ and $n_{e}$. Note that the current partitioning procedure gives $n_{i}=3 t / 2$ and $n_{e}=t$.

Furthermore, let $\vec{f}_{l} \in \mathbb{R}^{4}$ be the vector of normal components of the flux associated with the faces of a tetrahedron $\Delta_{l}$. In this section, we shall use the subscript $l$ for the vectors associated with the tetrahedron $\Delta_{l}$ and the superscript $i$ for their components.

Let $\overrightarrow{\hat{f}}=\left(\vec{f}^{e x t}, \vec{f}^{i n t}\right)$. Using the above notation, we write the inner product over the polyhedron $e$ as sum of the inner products over tetrahedra $\Delta_{l}$ :

$$
<\hat{M} \overrightarrow{\hat{f}}, \overrightarrow{\hat{h}}>=\sum_{l=1}^{t}<M_{\Delta_{l}} \vec{f}_{l}, \vec{h}_{l}>,
$$


where $M_{\Delta_{l}} \in \mathbb{R}^{4 \times 4}$ is defined in (4.2) and $\hat{M}$ is a symmetric positive definite matrix obtained by the standard assembling of matrices $M_{\Delta_{l}}, l=1 \ldots t$. This statement follows from the fact that the matrices $M_{\Delta_{l}}$ are symmetric positive definite and the matrix $\hat{M}$ is irreducible.

Our goal is to derive a discretization scheme using only one flux unknown per mesh face. Thus, we have to eliminate the temporary unknowns in favor of the original unknowns $f^{1}, f^{2}, \ldots, f^{s}$. The unknowns $\vec{f}{ }^{e x t}$ can be eliminated by setting them equal to the corresponding unknowns associated with the polyhedron faces. In other words, $f_{l}^{i}=f^{k}$ if the $i$-th face of $\Delta_{l}$ is a part of to the $k$-th face of $e$. In matrix form, this relation can be written as follows:

$$
\vec{f}^{e x t}=B_{e x t} \vec{f}
$$

where $B_{\text {ext }}$ is a matrix whose non-zero entries are equal to 1.

The unknowns $\vec{f}^{\text {int }}$ can be eliminated by requiring that the divergence over each tetrahedron is equal to the divergence over the polyhedron:

$$
\mathcal{D} I V_{l}^{h} \vec{f}_{l}=\mathcal{D} I V^{h} \vec{f}, \quad l=1 \ldots t .
$$

The definition of the discrete divergence operator (3.2) results in the following system of linear equations:

$$
\frac{1}{\left|V_{\Delta_{l}}\right|} \sum_{i=1}^{4} \alpha_{l}^{i}\left|A_{l}^{i}\right| f_{l}^{i}=\frac{1}{\left|V_{e}\right|} \sum_{k=1}^{s}\left|A^{k}\right| f^{k}, \quad l=1, \ldots, t
$$

where $\left|A_{l}^{i}\right|$ is area of the $i$-th face of $\Delta_{l}, \alpha_{l}^{i}=1$ if the normal defined on this face is the exterior normal for $\Delta_{l}$ and $\alpha_{l}^{i}=-1$ otherwise.

It is easy to show that this system of equations is linearly dependent. To make it linearly independent, we exclude one of these equations and consider a system of $t-1$ equations. Using (4.4), the reduced system can be written as follows:

$$
B_{i} \vec{f}^{i n t}=B_{e} \vec{f}
$$

where $B_{i} \in \mathbb{R}^{(t-1) \times n_{i}}$ and $B_{e} \in \mathbb{R}^{(t-1)^{6} \times s}$. The compatibility of this system is analyzed in the following lemma:

Lemma 4.1 The system (4.7) has a solution $\vec{f}^{\text {int }}$ for any vector $\vec{f}$.

The proof follows the proof of a similar result in [10]. In particular, it may be shown that the solution is not unique. To guarantee uniqueness, we impose one additional constraint; namely that the vector $\vec{f}^{i n t}$ is a solution of the following minimization problem:

$$
\min _{\vec{f}^{i n t}} \frac{1}{2}<\hat{M} \overrightarrow{\hat{f}}, \overrightarrow{\hat{f}}>\quad \text { subject to } \quad B_{i} \vec{f}^{i n t}=B_{e} \vec{f}
$$

The matrix $\hat{M}$ can be represented in block form following the partition of vector $\overrightarrow{\hat{f}}$ into vectors $\vec{f}^{\text {int }}$ and $\vec{f} \overrightarrow{e x t}^{x}$.

$$
\hat{M}=\left(\begin{array}{ll}
\hat{M}_{11} & \hat{M}_{12} \\
\hat{M}_{21} & \hat{M}_{22}
\end{array}\right)
$$


Since $\hat{M}$ is the symmetric matrix and the terms not involving $\vec{f}^{i n t}$ do not affect the minimizer of problem (4.8), we get the following problem:

$$
\min _{\vec{f}^{i n t}}\left[\frac{1}{2}<\hat{M}_{11} \vec{f}^{i n t}, \vec{f}^{i n t}>+<\hat{M}_{12} B_{e x t} \vec{f}, \vec{f}^{i n t}>\right] \quad \text { s.t. } \quad B_{i} \vec{f}^{i n t}=B_{e} \vec{f} .
$$

Lemma 4.2 The minimization problem (4.10) has the unique solution $\vec{f}^{\text {int }}$ for any vector $\vec{f}$ :

$$
\vec{f}^{i n t}=B_{\text {int }} \vec{f}
$$

where

$$
B_{i n t} \equiv \hat{M}_{11}^{-1}\left(-\hat{M}_{12} B_{\text {ext }}+B_{i}\left[B_{i} \hat{M}_{11}^{-1} B_{i}^{T}\right]^{-1}\left[B_{e}+B_{i} \hat{M}_{11}^{-1} \hat{M}_{12} B_{\text {ext }}\right]\right) .
$$

Proof. The constrained minimization problem can be written, using a Lagrange multiplier $\vec{\lambda}$, as follows:

$$
\left[\begin{array}{cc}
\hat{M}_{11} & -B_{i}^{T} \\
B_{i} & 0
\end{array}\right]\left[\begin{array}{c}
\vec{f}^{i n t} \\
\vec{\lambda}
\end{array}\right]=\left[\begin{array}{c}
-\hat{M}_{12} B_{e x t} \vec{f} \\
B_{e} \vec{f}
\end{array}\right]
$$

Solving the first set of equations for $\vec{f}^{i n t}$, we get

$$
\vec{f}^{i n t}=\hat{M}_{11}^{-1}\left(-\hat{M}_{12} B_{e x t} \vec{f}+B_{i}^{T} \vec{\lambda}\right)
$$

This result can be substituted into the second set of equations which can be solved for $\vec{\lambda}$ to produce

$$
\vec{\lambda}=\left[B_{i} \hat{M}_{11}^{-1} B_{i}^{T}\right]^{-1}\left[B_{e}+B_{i} \hat{M}_{11}^{-1} \hat{M}_{12} B_{e x t}\right] \vec{f}
$$

Substituting this result into the formula for $\vec{f}^{i n t}$, we prove the assertion of the lemma.

Using (4.4) and Lemma 4.2, we may rewrite the inner product (4.3) as follows:

$$
<M \vec{f}, \vec{h}\rangle \equiv\left\langle\left(\begin{array}{ll}
\hat{M}_{11} & \hat{M}_{12} \\
\hat{M}_{21} & \hat{M}_{22}
\end{array}\right)\left(\begin{array}{c}
B_{\text {int }} \vec{f} \\
B_{\text {ext }} \vec{f}
\end{array}\right),\left(\begin{array}{c}
B_{\text {int }} \vec{h} \\
B_{\text {ext }} \vec{h}
\end{array}\right)\right\rangle .
$$

It is obvious that $M$ is a symmetric positive definite matrix. Indeed, it is the restriction of the symmetric positive definite matrix $\hat{M}$ to the subspace of vectors $\left(B_{i n t} \vec{h}, B_{\text {ext }} \vec{h}\right)^{T}$. Thus, it may be used to generate the inner product on $\mathbb{X}^{h}$. The explicit formula for matrix $M$ is as follows:

$$
M=\hat{B}_{\text {ext }}^{T} \hat{M}_{22} B_{\text {ext }}+B_{i n t}^{T} \hat{M}_{11} B_{\text {int }}+B_{\text {ext }}^{T} \hat{M}_{21} B_{\text {int }}+B_{i n t}^{T} \hat{M}_{12} B_{\text {ext }} .
$$




\section{Solution algorithm}

Let us collect the face-based flux unknowns and cell-centered pressure unknowns into two global vectors

$$
\overrightarrow{f_{A}}=\left(\vec{f}_{1}^{T}, \vec{f}_{2}^{T}, \ldots, \vec{f}_{N}^{T}\right)^{T}, \quad \text { and } \quad \vec{p}_{0}=\left(p_{1}^{0}, p_{2}^{0}, \ldots, p_{N}^{0}\right)^{T},
$$

respectively. Taking into account continuity conditions (3.7), we collect unique facebased pressure unknowns into a global vector $\vec{p}_{A}$. The size of this vector is equal to the number of mesh faces.

Then, the system of discrete equations (3.6), flux continuity conditions (3.7), and boundary conditions (3.8) and (3.9) can be written in the matrix form:

$$
\mathcal{A}\left(\begin{array}{l}
\vec{f}_{A} \\
\vec{p}_{0} \\
\vec{p}_{A}
\end{array}\right)=\left(\begin{array}{l}
\vec{g}_{D} \\
\vec{Q}_{0} \\
\vec{g}_{R}
\end{array}\right),
$$

with the saddle point matrix

$$
\mathcal{A}=\left(\begin{array}{ccc}
M & B & C \\
B^{T} & -D & 0 \\
C^{T} & 0 & -\Sigma
\end{array}\right)
$$

where

$$
M=\left(\begin{array}{ccc}
M_{1} & & 0 \\
& \ddots & \\
0 & & M_{N}
\end{array}\right)
$$

is the block diagonal matrix with symmetric positive definite blocks on the diagonal, $D$ is the diagonal positive definite or semi-definite matrix and $\Sigma$ is the diagonal positive semi-definite matrix.

Lemma 5.1 Using $O(N)$ arithmetical operations the system (5.1) can be transformed into the following system:

$$
S \vec{p}_{A}=\vec{h}_{A}
$$

where

$$
S=C^{T} M^{-1} C-C^{T} M^{-1} C B\left(B^{T} M^{-1} B+D\right)^{-1} B^{T} M^{-1} C+\Sigma
$$

is the symmetric matrix and

$$
\vec{h}_{A}=C^{T} M^{-1} \vec{g}_{D}-C^{T} M^{-1} B\left(B^{T} M^{-1} B+D\right)^{-1}\left(\vec{Q}_{0}+B^{T} M^{-1} \vec{g}_{D}\right)-\vec{g}_{R} .
$$

Proof. It is pertinent to note that the primary variables $\vec{f}_{i}$ and $p_{i}^{0} i=1, \ldots N$ are only connected within a single polyhedron. So we can easily exclude the unknowns:

$$
\overrightarrow{f_{A}}=M^{-1}\left(\vec{g}_{D}-C \vec{p}_{A}-B \vec{p}_{0}\right)
$$

and

$$
\vec{p}_{0}=\left(B^{T} M^{-1} B+D\right)^{-1}\left(\vec{g}_{D}-C \vec{p}_{A}-B \vec{p}_{0}\right)
$$


The structure of matrices $M, B$ and $D$ implies that matrix $B^{T} M^{-1} B+D$ is diagonal and so it is easily invertible. This implies optimal arithmetical complexity. The other assertions of the lemma follow by substituting (5.3) and (5.4) in the last equation of system (5.1).

The matrix $S$ is positive definite except the case of the Neumann boundary condition on $\partial \Omega$ when it is positive semi-definite. Thus, we can apply a preconditioned conjugate gradient (PCG) method for solving system (5.2). After that, the primary unknowns $\vec{p}_{0}$ and $\overrightarrow{f_{A}}$ can be recovered locally, element-by-element.

The matrix $\mathcal{A}$ has a very useful representation, namely

$$
\mathcal{A}=\sum_{i=1}^{N} \mathcal{N}_{i} \mathcal{A}_{i} \mathcal{N}_{i}^{T}
$$

where

$$
\mathcal{A}_{i}=\left(\begin{array}{ccc}
M_{i} & B_{i} & C_{i} \\
B_{i}^{T} & -D_{i} & 0 \\
C_{i}^{T} & 0 & -\Sigma_{i}
\end{array}\right)
$$

is the local saddle point matrix for the polyhedron $e_{i}$ and $\mathcal{N}_{i}$ is the corresponding assembling matrix. To show the important properties of matrices $M_{i}, B_{i}$ and $C_{i}$ and $D_{i}$, we consider an internal polyhedron $e_{i}$, i.e. $e_{i} \cap \partial \Omega_{h}=\emptyset$. In this case $M_{i}$ is a symmetric positive definite matrix,

$$
B_{i}=\left(-\left|A_{i}^{1}\right|,-\left|A_{i}^{2}\right|, \ldots,-\left|A_{i}^{s_{i}}\right|\right)^{T}, \quad C_{i}=\operatorname{diag}\left\{\left|A_{i}^{1}\right|,\left|A_{i}^{2}\right|, \ldots,\left|A_{i}^{s_{i}}\right|\right\}
$$

and $D_{i}=c_{i}\left|V_{e_{i}}\right|$.

\section{$6 \quad$ Numerical experiments}

In this section, we present computational results which demonstrate accuracy of the mimetic discretization, its flexibility, and efficiency of the solution method.

\subsection{Implementation issues}

To solve system (5.2) we apply the PCG method with the algebraic multigrid preconditioner discussed in [18]. This method is applicable to arbitrary matrix stencils; however, its theoretical justification is limited to M-matrices.

We investigate the convergence of pressure unknowns in the following norms:

$$
\varepsilon_{\infty}^{p}=\max _{1 \leq i \leq N}\left|p_{i}^{e x}-p_{i}^{0}\right| \quad \text { and } \quad \varepsilon_{2}^{p}=\left[\sum_{i=1}^{N}\left(p_{i}^{e x}-p_{i}^{0}\right)^{2}\left|V_{e_{i}}\right|\right]^{1 / 2},
$$

where $p_{i}^{e x}$ is the exact pressure value at the center of mass of the polyhedron $e_{i}$.

For the flux unknowns, we use two similar norms:

$$
\varepsilon_{\infty}^{f}=\max _{1 \leq i \leq N}\left\|\overrightarrow{f_{i}^{e x}}-\overrightarrow{f_{i}}\right\|_{\infty} \quad \text { and } \quad \varepsilon_{2}^{f}=\left[\sum_{i=1}^{N}<M_{i}\left(\overrightarrow{f_{i}^{e x}}-\overrightarrow{f_{i}}\right),\left(\overrightarrow{f_{i}^{e x}}-\overrightarrow{f_{i}}\right)>\right]^{1 / 2}
$$


where the components of vector $\overrightarrow{f_{i}^{e x}}$ are normal components of the exact flux averaged over the faces of polyhedron $e_{i}$ and $\|\cdot\|_{\infty}$ is the maximal norm in the Euclidean space. Note that $\varepsilon_{2}^{p}$ and $\varepsilon_{2}^{f}$ are mesh norms equivalent to $L_{2}$-norms in the corresponding continuous spaces.

\subsection{Smooth meshes}

In the first group of numerical tests, we investigate the accuracy of the mimetic discretization on smooth meshes. As the example of a smooth mesh, we consider the mesh obtained by a smooth ( $C^{2}$-regular) mapping of a uniform cubic mesh. Let us consider a uniform partition of the unit cube $[0,1]^{3}$ and the following mapping:

$$
\left(\begin{array}{l}
\tilde{x} \\
\tilde{y} \\
\tilde{z}
\end{array}\right)=\left(\begin{array}{l}
x \\
y \\
z
\end{array}\right)+0.1\left(\begin{array}{l}
1 \\
1 \\
1
\end{array}\right) \sin (2 \pi x) \sin (2 \pi y) \sin (2 \pi z) .
$$

Since the Jacobian of this mapping is positive, the resulting smooth mesh, $\Omega_{h}$, does not contain degenerate polyhedra. Note that most of the mesh faces are curved, so the convergence theory developed in [4] can not be applied here. However, the deviation from flat faces is of order $h^{2}$ which is typical for smooth meshes. An example of $\Omega_{h}$ is presented in Fig. 3 where we show the internal mesh structure. We visualize the polyhedra whose centers are inside domain $(0,1)^{3} \backslash[0.25,1]^{3}$.

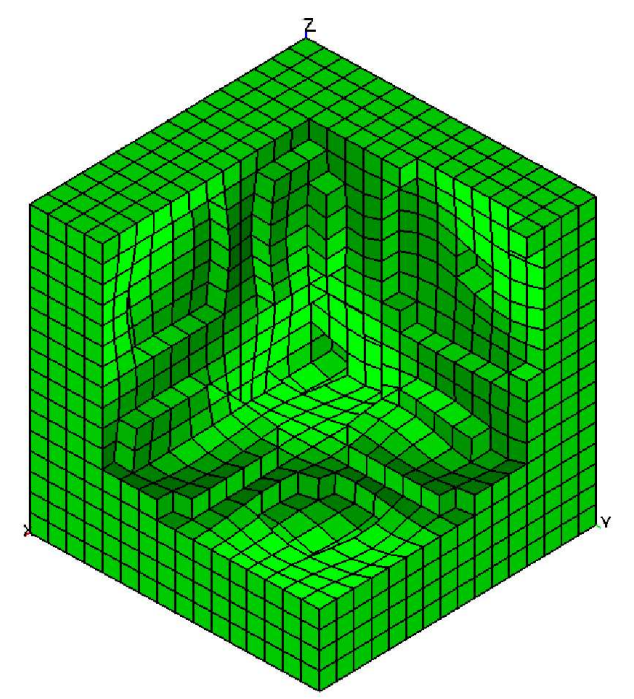

Figure 3: An example of a smooth mesh.

Now, we consider a diffusion problem of type (2.1), with a non-homogeneous Dirichlet boundary condition on $\partial \Omega$. Let $c(\mathbf{x}) \equiv 0, K(\mathbf{x}) \equiv 1$ and the exact solution be

$$
p^{e x}(x, y, z)=x^{2} y^{3} z+3 x \sin (y z)
$$

The convergence results are shown in Table 1 where $h$ denotes the size of a cubic cell in the original uniform partition of the computational domain. We use a linear 
regression algorithm to estimate convergence rates. The decrease in both errors $\varepsilon_{p}^{2}$ and $\varepsilon_{f}^{2}$ approaches the optimal rate which is 2 . This is clear when looking at the errors in rows corresponding to $1 / h=32$ and $1 / h=64$.

\begin{tabular}{c||cc|cc}
$1 / \mathrm{h}$ & $\varepsilon_{\infty}^{p}$ & $\varepsilon_{2}^{p}$ & $\varepsilon_{\infty}^{f}$ & $\varepsilon_{2}^{f}$ \\
\hline 8 & $1.037 \mathrm{e}-2$ & $2.250 \mathrm{e}-3$ & $1.873 \mathrm{e}-1$ & $4.053 \mathrm{e}-2$ \\
16 & $5.017 \mathrm{e}-3$ & $7.483 \mathrm{e}-4$ & $7.211 \mathrm{e}-2$ & $1.089 \mathrm{e}-2$ \\
32 & $1.669 \mathrm{e}-3$ & $2.027 \mathrm{e}-4$ & $2.191 \mathrm{e}-2$ & $2.707 \mathrm{e}-3$ \\
64 & $4.753 \mathrm{e}-4$ & $5.177 \mathrm{e}-5$ & $5.987 \mathrm{e}-3$ & $6.628 \mathrm{e}-4$ \\
\hline rate & 1.49 & 1.82 & 1.66 & 1.98
\end{tabular}

Table 1: Discretization errors on smooth meshes for $K=1$.

Now we change the setup of the previous experiment in order to analyze the influence of a full diffusion tensor on the convergence rates. Let $K$ be as follows:

$$
K(x, y, z)=\left(\begin{array}{ccc}
y^{2}+z^{2}+1 & x y & x z \\
x y & x^{2}+z^{2}+1 & y z \\
x z & y z & x^{2}+y^{2}+1
\end{array}\right)
$$

This tensor is symmetric and positive definite for arbitrary $\mathbf{x}$. The exact solution and the meshes are as in the previous experiment. The convergence results are presented in Table 2. Again, the decrease in both errors $\varepsilon_{2}^{p}$ and $\varepsilon_{2}^{f}$ approaches the optimal rate. We observed in many other experiments that the full diffusion tensor does not affect asymptotic convergence rates.

\begin{tabular}{c||cc|cc}
$1 / \mathrm{h}$ & $\varepsilon_{\infty}^{p}$ & $\varepsilon_{2}^{p}$ & $\varepsilon_{\infty}^{f}$ & $\varepsilon_{2}^{f}$ \\
\hline 8 & $2.059 \mathrm{e}-2$ & $4.359 \mathrm{e}-3$ & $8.974 \mathrm{e}-1$ & $1.011 \mathrm{e}-1$ \\
16 & $5.552 \mathrm{e}-3$ & $1.129 \mathrm{e}-3$ & $3.008 \mathrm{e}-1$ & $2.826 \mathrm{e}-2$ \\
32 & $1.402 \mathrm{e}-3$ & $2.875 \mathrm{e}-4$ & $8.572 \mathrm{e}-2$ & $7.278 \mathrm{e}-3$ \\
64 & $3.514 \mathrm{e}-4$ & $7.229 \mathrm{e}-5$ & $2.999 \mathrm{e}-2$ & $1.821 \mathrm{e}-3$ \\
\hline rate & 1.96 & 1.97 & 1.65 & 1.93
\end{tabular}

Table 2: Discretization errors on smooth meshes for the full tensor $K$.

\subsection{Randomly perturbed meshes}

The next set of tests addresses the convergence of the mimetic discretization on randomly perturbed meshes. Such a mesh is obtained by random distortion of positions of mesh points in a uniform cubic mesh. The new positions are determined by the following formulas:

$$
\tilde{x}=x+\xi_{x} h, \quad \tilde{y}=y+\xi_{y} h, \quad \tilde{z}=z+\xi_{z} h
$$

where $\xi_{x}, \xi_{y}, \xi_{z}$ are random numbers between -0.3 and 0.3 and $h$ is the mesh step size. In other words each mesh point is randomly moved in a cube of size $0.6 h$ which is centered 
at the point and whose edges are aligned with the coordinate axes. It is pertinent to note that in many publications related to convergence of discrete methods on general meshes, the authors consider a sequence of meshes obtained by uniform refinement of a coarse randomly perturbed mesh. In this case, the mimetic discretization results in optimal convergence rates. Sequences of true randomly perturbed meshes are more general and more difficult for convergence analysis.

Applying the distortion described above, we obtain polyhedra with strongly curved faces. To the best of our knowledge, there is no locally conservative discretization method which uses one flux unknown per mesh face, one pressure unknown per mesh element and converges on randomly perturbed meshes (see also Table 3). Therefore, we propose to use more than one flux unknown per strongly curved face. We use our definition of a curved face to replace it with a set of triangular facets. As shown in Fig. 4, a distorted cube is transformed into a polyhedron with 24 planar triangular faces. Finally, approximating all curved faces, we obtain a mesh like one shown in Fig. 5. The mimetic discretization described above will use one flux unknown per each triangular face and one pressure unknown per each 24 -face polyhedron. With respect to the original hexahedral mesh, we shall refer to this discretization as the discretization with 4 flux unknowns per mesh face.
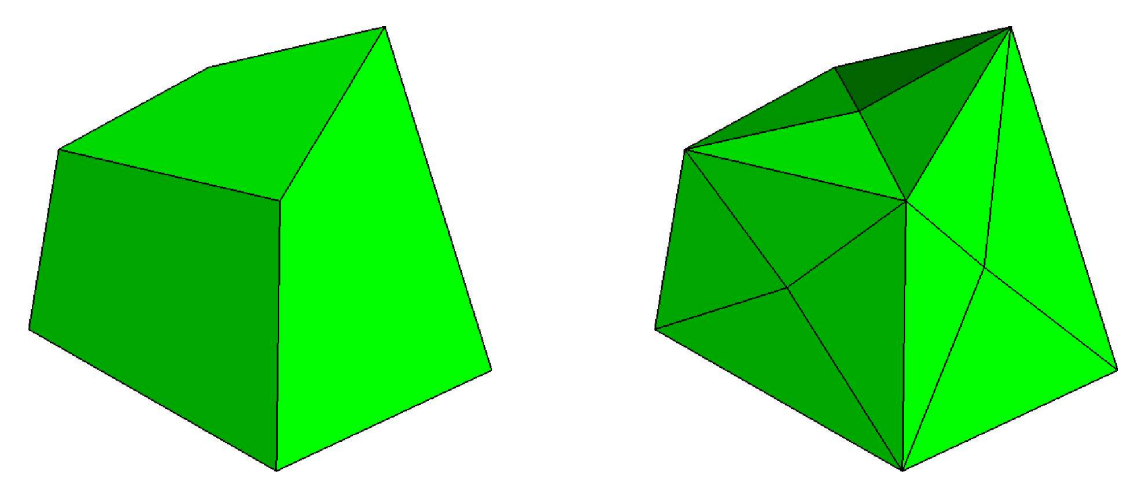

Figure 4: Transformation of a cube to a polyhedron with 24 planar faces.

We consider the same diffusion problem as in the previous set of experiments. The computational results are shown in Tables 3 and 4 . For the case of 4 flux unknowns per mesh face, the convergence rates are close to optimal. Note that there is no superconvergence results for the flux variable on randomly perturbed meshes. With only one flux unknown per curved face, we lose convergence for both pressure and flux variables (see Table 3).

\subsection{Meshes with irregular-shaped polyhedra}

In this subsection, we analyze convergence rate and robustness of the mimetic discretization on meshes with irregular-shaped elements. Following the analysis in [10], we anticipate that in most practically important cases, the irregular-shaped elements will not present computational problems. 


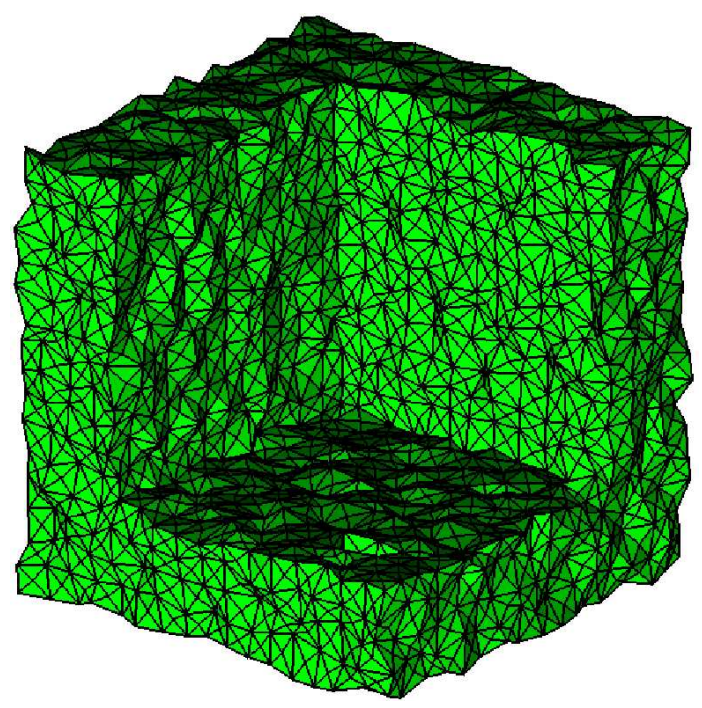

Figure 5: An example of a randomly perturbed mesh.

\begin{tabular}{c||cc|cc}
$1 / \mathrm{h}$ & $\varepsilon_{\infty}^{p}$ & $\varepsilon_{2}^{p}$ & $\varepsilon_{\infty}^{f}$ & $\varepsilon_{2}^{f}$ \\
\hline 8 & $4.121 \mathrm{e}-2$ & $1.115 \mathrm{e}-2$ & $2.993 \mathrm{e}-0$ & $3.132 \mathrm{e}-1$ \\
16 & $2.165 \mathrm{e}-2$ & $6.861 \mathrm{e}-3$ & $2.803 \mathrm{e}-0$ & $2.297 \mathrm{e}-1$ \\
32 & $1.829 \mathrm{e}-2$ & $5.756 \mathrm{e}-3$ & $4.242 \mathrm{e}-0$ & $2.112 \mathrm{e}-1$ \\
64 & $1.750 \mathrm{e}-2$ & $5.504 \mathrm{e}-3$ & $4.544 \mathrm{e}-0$ & $2.094 \mathrm{e}-1$
\end{tabular}

Table 3: Discretization errors on randomly perturbed meshes using 1 flux unknown per curved face.

Let us modify the uniform cubic partition of $\Omega=(0,1)^{3}$ with mesh step size $h$ in such a way that irregular-shaped polyhedra appear. We assume that a mesh point with the logical coordinates $(i, j, k)$ has the physical coordinates $(i h, j h, k h), i, j, k=0, \ldots 1 / h$. Let us introduce a distortion parameter $\alpha \in[0,0.5]$ such that the smaller values of $\alpha$ correspond to elements with less shape regularity. Then, for all odd $i$ and $k$ and for any $j$ the coordinates of point $(i h, j h, k h)$ are changed to $(i h-\alpha h, j h, k h-(1-\alpha) h)$. The example of such a modification is shown in Fig. 6.

As we can see in Fig. 6, the neighboring polyhedra have a common face with relatively small area. Moreover, the left polyhedron has two faces with $2 \mathrm{D}$ angle between them

\begin{tabular}{c||cc|cc}
$1 / \mathrm{h}$ & $\varepsilon_{\infty}^{p}$ & $\varepsilon_{2}^{p}$ & $\varepsilon_{\infty}^{f}$ & $\varepsilon_{2}^{f}$ \\
\hline 8 & $1.160 \mathrm{e}-2$ & $2.844 \mathrm{e}-3$ & $7.957 \mathrm{e}-1$ & $9.861 \mathrm{e}-2$ \\
16 & $3.088 \mathrm{e}-3$ & $7.140 \mathrm{e}-4$ & $6.000 \mathrm{e}-1$ & $4.537 \mathrm{e}-2$ \\
32 & $1.068 \mathrm{e}-3$ & $1.790 \mathrm{e}-4$ & $3.494 \mathrm{e}-1$ & $2.246 \mathrm{e}-2$ \\
\hline rate & 1.72 & 1.99 & 0.59 & 1.07
\end{tabular}

Table 4: Discretization errors on randomly perturbed meshes using 4 flux unknowns per curved face. 

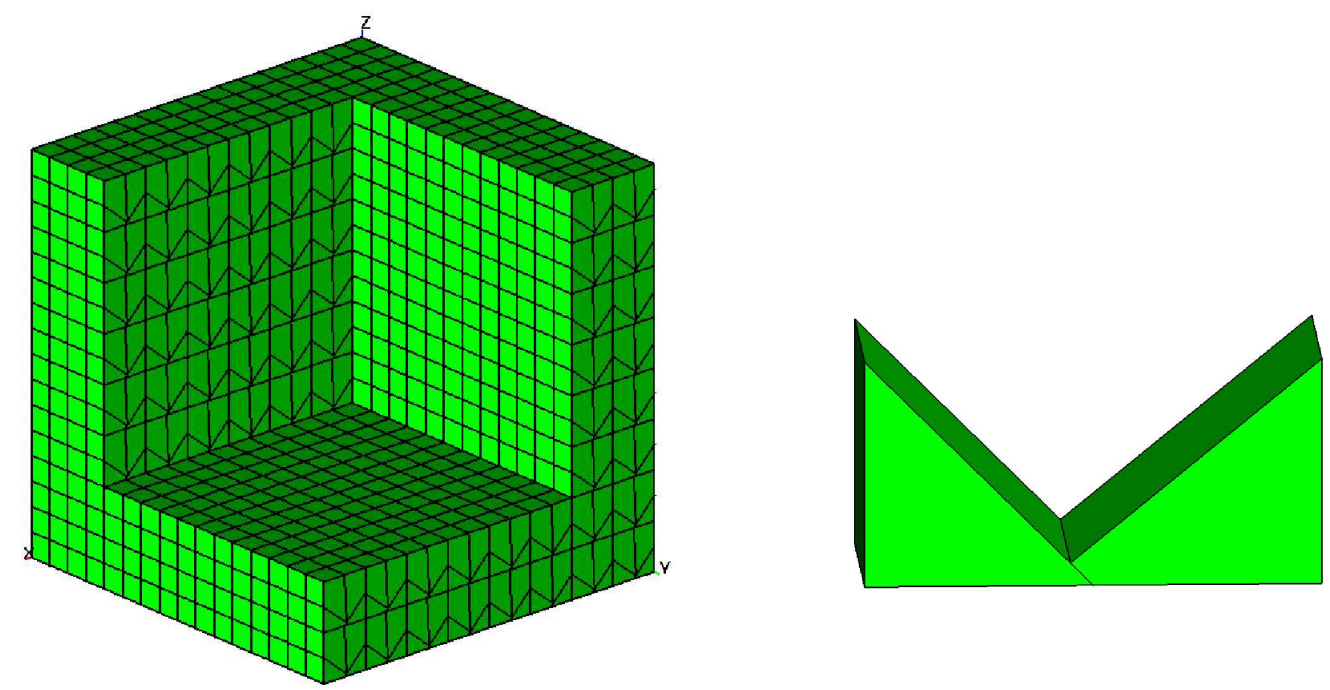

Figure 6: The mesh with irregular-shaped polyhedra (left) and two typical neighboring elements.

equal to $\pi$.

We consider the same diffusion problem as in the previous set of experiments. But now we investigate the dependence of convergence rates on shape regularity of mesh elements. The computational results are presented in Table 5 where $\sigma$ is the ratio of the maximal face area to the minimal one. Thus, $\sigma=\infty$ means that a hexahedron is transformed into a pentahedron.

\begin{tabular}{c||c|c|c|c|c|c}
\multicolumn{1}{c||}{$1 / \mathrm{h}$} & \multicolumn{3}{c|}{$\varepsilon_{2}^{p}$} & \multicolumn{3}{c}{$\varepsilon_{2}^{f}$} \\
\hline \multicolumn{1}{l}{} & $\alpha=0.1$ & $\alpha=0.01$ & $\alpha=0.0$ & $\alpha=0.1$ & $\alpha=0.01$ & $\alpha=0.0$ \\
& $\sigma=700$ & $\sigma=7000$ & $\sigma=\infty$ & $\sigma=700$ & $\sigma=7000$ & $\sigma=\infty$ \\
\hline 8 & $5.386 \mathrm{e}-3$ & $5.858 \mathrm{e}-3$ & $5.944 \mathrm{e}-3$ & $1.280 \mathrm{e}-1$ & $1.459 \mathrm{e}-1$ & $1.495 \mathrm{e}-1$ \\
16 & $1.358 \mathrm{e}-3$ & $1.484 \mathrm{e}-3$ & $1.506 \mathrm{e}-3$ & $6.101 \mathrm{e}-2$ & $6.974 \mathrm{e}-2$ & $7.159 \mathrm{e}-2$ \\
32 & $3.407 \mathrm{e}-4$ & $3.731 \mathrm{e}-4$ & $3.789 \mathrm{e}-4$ & $2.982 \mathrm{e}-2$ & $3.407 \mathrm{e}-2$ & $3.501 \mathrm{e}-2$ \\
64 & $8.528 \mathrm{e}-5$ & $9.353 \mathrm{e}-5$ & $9.497 \mathrm{e}-5$ & $1.474 \mathrm{e}-2$ & $1.683 \mathrm{e}-2$ & $1.731 \mathrm{e}-2$ \\
\hline rate & 1.99 & 1.99 & 1.99 & 1.04 & 1.04 & 1.04
\end{tabular}

Table 5: Discretization errors on meshes with irregular-shaped polyhedra.

The numerical results presented in Table 5 verify that decrease of shape regularity of mesh elements does not affect convergence of the mimetic discretization.

\subsection{Non-matching meshes}

In the next group of numerical tests, we consider non-matching cubic meshes. Let $\Omega_{1}=(0,1)^{3}, \Omega_{2}=(1,2) \times(0,1)^{2}$ and $\Omega=\Omega_{1} \cup \Omega_{2}$. We consider the same diffusion problem as in the previous experiment, only now in the bigger domain. 
Let $\Omega_{1, h}$ and $\Omega_{2, h}$ be uniform cubic meshes with mesh sizes $h_{1}$ and $h_{2}$, respectively. In order to obtain the conformal partition of $\Omega$, we introduce additional faces and edges on the non-matching interface. One example of a conformal partition of $\Omega$ and the modified interface between subdomains $\Omega_{1}$ and $\Omega_{2}$ are shown in Fig. 7 .
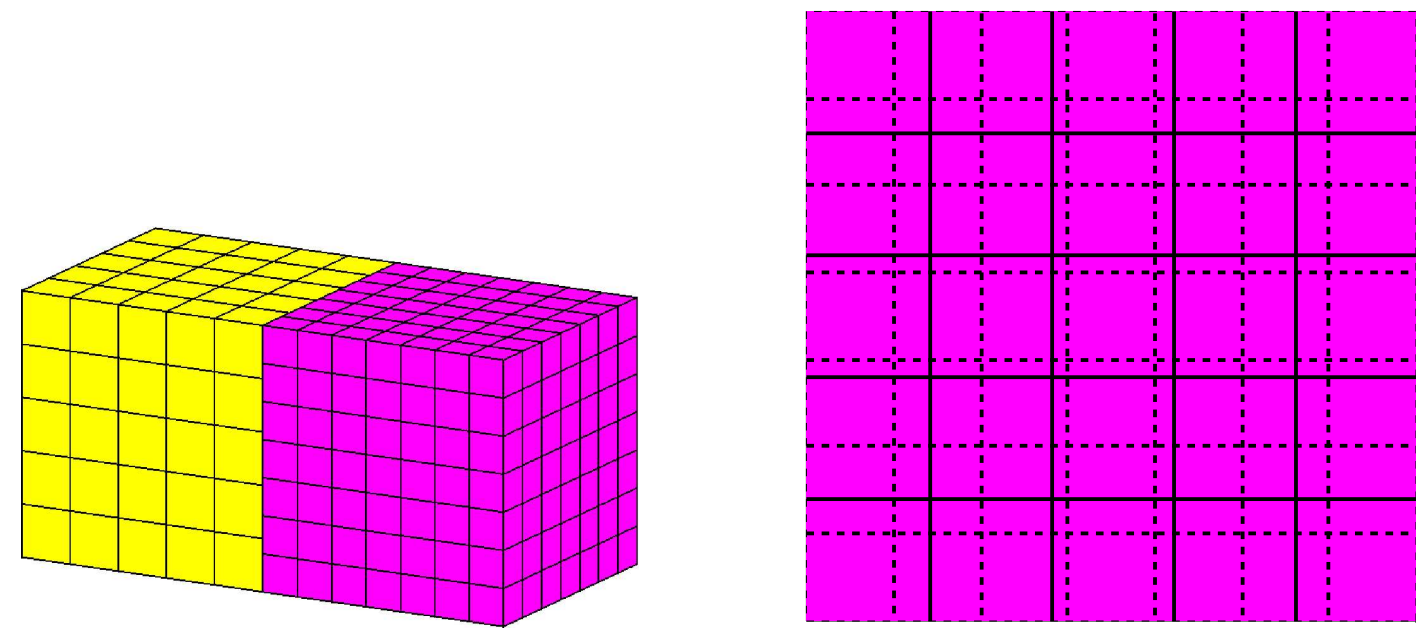

Figure 7: An example of non-matching meshes with $h_{1}=1 / 5$ and $h_{2}=1 / 7$.

In the case of non-matching meshes, the interface elements have many more faces then other mesh elements and their shape regularity may be very poor. For example, one of the interface polyhedra shown in Fig. 7 has 14 face. However, it was shown in the previous subsection that the presence of degenerate elements does not affect the rate of convergence. The numerical results presented in Table 6 once again confirm this statement. The superconvergence rate of 1.5 for the flux variable is observed in other lower order discretization methods, e.g., in the mortar finite element method with the lowest order Raviart-Thomas elements [2].

\begin{tabular}{cc||cc|cc}
$1 / h_{1}$ & $1 / h_{2}$ & $\varepsilon_{\infty}^{p}$ & $\varepsilon_{2}^{p}$ & $\varepsilon_{\infty}^{f}$ & $\varepsilon_{2}^{f}$ \\
\hline 7 & 5 & $1.126 \mathrm{e}-3$ & $1.604 \mathrm{e}-4$ & $4.61 \mathrm{e}-2$ & $3.5029 \mathrm{e}-3$ \\
14 & 10 & $3.206 \mathrm{e}-4$ & $4.078 \mathrm{e}-5$ & $3.232 \mathrm{e}-2$ & $1.144 \mathrm{e}-3$ \\
28 & 20 & $8.627 \mathrm{e}-5$ & $1.025 \mathrm{e}-5$ & $2.142 \mathrm{e}-2$ & $3.837 \mathrm{e}-4$ \\
\hline rate & & 1.853 & 1.983 & 0.552 & 1.595
\end{tabular}

Table 6: Discretization errors on non-matching meshes

\subsection{Flow through a system containing an impermeable pipe}

Let us consider the unit cube $\Omega=(0,1)^{3}$ and a pipe imbedded in it. We assume that the pipe profile in any $y z$-plane is a circle with constant radius $r$. The circle centers form a curve $\phi(t):\{x=x(t), y=y(t), z=z(t)\}$ whose starting and ending points are in the planes $x=0$ and $x=1$, respectively. This problem was selected to demonstrate 
capability of the mimetic discretization to produce qualitatively accurate results. Since the problem does not have an analytical solution, it is extremely difficult to quantify accuracy of the result.

Let us consider a uniform cubic partition of $\Omega$. We modify this partition in such a way that the faces of the new partition, $\Omega_{h}$, approximate the pipe surface. In order to do so, we use an algorithm for building locally fitted meshes. Since the resulting mesh may have degenerate and non-convex elements, we use the following simple algorithm: if the pipe surface intersects a cubic element and the center of mass of this element is inside the pipe than the element vertices located outside the pipe are moved to the pipe surface. Otherwise, if the center of mass is outside the pipe then the element vertices located inside the pipe are moved to the pipe surface. The points are moved only in $y z$-planes. An example of a locally fitted mesh is shown in Fig. 8.
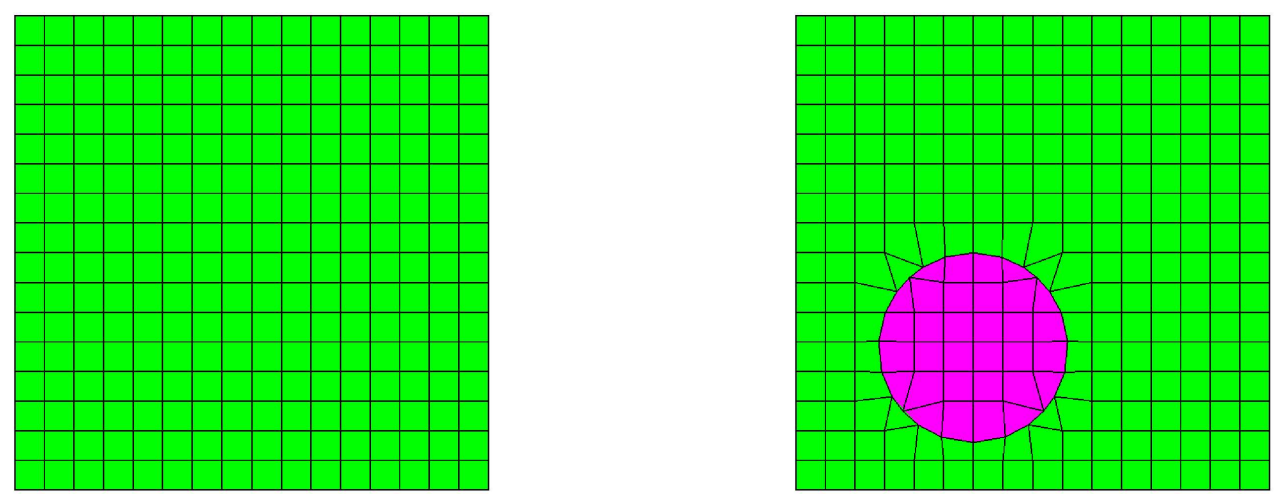

Figure 8: A cut of the original and locally fitted meshes by a yz-plane.

Now, we consider the diffusion equation (2.1) with $c(\mathbf{x}) \equiv 0$ and $Q(\mathbf{x}) \equiv 0$. We impose the non-homogeneous Dirichlet boundary condition $g_{D}(\mathbf{x})=1$ on the plane $x=0$, the homogeneous Dirichlet boundary condition on the plane $x=1$ and the homogeneous Neumann boundary condition on the remaining part of $\partial \Omega$.

The diffusion tensor $K$ is uniform and isotropic everywhere in the computational domain $(K=1)$ except in the pipe where it is set such that the component parallel to the local pipe orientation $\left(\boldsymbol{k}_{\|}\right)$is equal to 0.1 and other two components perpendicular to the pipe orientation $\left(\boldsymbol{k}_{\perp}^{1}\right.$ and $\left.\boldsymbol{k}_{\perp}^{2}\right)$ are equals to 0.001 . Inside the pipe, $K$ is the full tensor depending on the space coordinate $\mathbf{x}$. The vector $\boldsymbol{k}_{\|}$coincides with the tangent vector $\boldsymbol{t}$ to the curve $t \rightarrow \phi(t)$ :

$$
\boldsymbol{k}_{\|}=\boldsymbol{t}=\boldsymbol{\phi}(t)^{\prime}
$$

Vectors $\boldsymbol{k}_{\perp}^{1}$ and $\boldsymbol{k}_{\perp}^{2}$ coincide with the normal vector $\boldsymbol{n}$ and the binormal vector $\boldsymbol{b}$ to the curve $t \rightarrow \phi(t)$, respectively:

$$
\boldsymbol{k}_{\perp}^{1}=\boldsymbol{n}=\frac{\boldsymbol{\phi}(t)^{\prime \prime}}{\left|\boldsymbol{\phi}(t)^{\prime}\right|} \quad \text { and } \quad \boldsymbol{k}_{\perp}^{2}=\boldsymbol{b}=[\boldsymbol{t} \times \boldsymbol{n}] .
$$


Then, the diffusion tensor inside the pipe is calculated by the following formula:

$$
K=\mathbf{T}^{*}\left(\begin{array}{ccc}
0.1 & 0 & 0 \\
0 & 0.001 & 0 \\
0 & 0 & 0.001
\end{array}\right) \mathbf{T}, \quad \mathbf{T}=\left(\begin{array}{ccc}
\boldsymbol{t}_{x} & \boldsymbol{n}_{x} & \boldsymbol{b}_{x} \\
\boldsymbol{t}_{y} & \boldsymbol{n}_{y} & \boldsymbol{b}_{y} \\
\boldsymbol{t}_{z} & \boldsymbol{n}_{z} & \boldsymbol{b}_{z}
\end{array}\right)
$$

Fig. 9 displays streamlines of the calculated flux function in the case where $\phi(t)=$ $\left\{x=t, y=0.75-0.4 t^{2}, z=0.3\right\}$. The locally adapted mesh has both non-convex and almost degenerate elements. However, none of the streamlines intersects the pipe boundary.

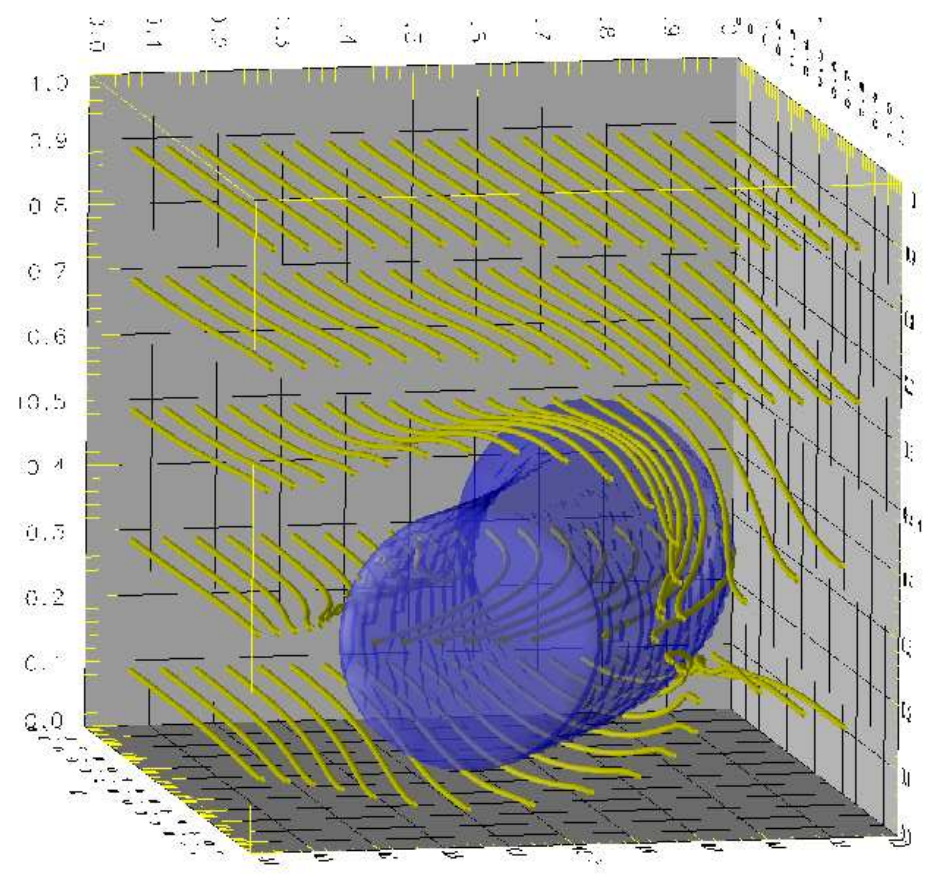

Figure 9: The streamlines of the calculated flux function.

\section{Acknowledgments}

The authors thank Dr. Rao Garimella (LANL) for his assistance in generating polyhedral meshes and Prof. Yuri Kuznetsov (University of Houston) for many valuable comments.

\section{References}

[1] I. Aavatsmark. An introduction to multipoint flux approximations for quadrilateral grids. Comp. Geosciences, 6:405-432, 2002.

[2] T. Arbogast, L. Cowsar, M. Wheeler, and I. Yotov. Mixed finite element methods on non-matching multiblock grids. SIAM J. Numer. Anal., 37:1295-1315, 2000. 
[3] T. Austin, J. Morel, J. Moulton, and M. Shashkov. Mimetic preconditioners for mixed discretizations of the diffusion equation. Technical Report LA-UR-01-807, Los Alamos National Laboratory, 2004. www.ima.umn.edu/talks/workshops/5-1115.2004/moulton/moulton.pdf.

[4] F. Brezzi, K. Lipnikov, and M. Shashkov. Convergence of mimetic finite difference method for diffusion problems on polyhedral meshes. SIAM J. Numer. Anal., 2005. to appear.

[5] J. Campbell and M. Shashkov. A tensor artificial viscosity using a mimetic finite difference algorithm. J. Comput. Phys., 172:739-765, 2001.

[6] P. Grisvard. Elliptic Problems in Nonsmooth domains. Pitman, London, 1985.

[7] J. Hyman, J. Morel, M. Shashkov, and S. Steinberg. Mimetic finite difference methods for diffusion equations. Comp. Geosciences, 6(3-4):333-352, 2002.

[8] J. Hyman and M. Shashkov. Mimetic discretizations for Maxwell's equations and the equations of magnetic diffusion. Progress in Electromagnetic Research, 32:89$121,2001$.

[9] J. Hyman, M. Shashkov, and S. Steinberg. The numerical solution of diffusion problems in strongly heterogeneous non-isotropic materials. J. Comput. Phys., 132:130-148, 1997.

[10] Y. Kuznetsov, K. Lipnikov, and M. Shashkov. Mimetic finite difference method on polygonal meshes for diffusion-type problems. Comp. Geosciences, 2004. in press.

[11] Y. Kuznetsov and S. Repin. New mixed finite element method on polygonal and polyhedral meshes. Russ. J. Numer. Anal. Math. Modelling, 18(3):261-278, 2003.

[12] Y. Kuznetsov and S. Repin. Convergence analysis and error estimates for mixed finite element method on distrted meshes. J. Numer. Math., 13(1):33-51, 2005.

[13] K. Lipnikov, J. Morel, and M. Shashkov. Mimetic finite difference methods for diffusion equations on non-orthogonal non-conformal meshes. J. Comput. Phys., 199, 2004.

[14] L. Margolin, M. Shashkov, and P. Smolarkiewicz. A discrete operator calculus for finite difference approximations. Comput. Meth. Appl. Mech. Engrg., 187:365-383, 2000 .

[15] I. Mishev. Nonconforming finite volume methods. Comp. Geosciences, 6:253-268, 2002.

[16] J. Morel, R. Roberts, and M. Shashkov. A local support-operators diffusion discretization scheme for quadrilateral $r-z$ meshes. J. Comput. Phys., 144:17-51, 1998. 
[17] P. Raviart and J.-M. Thomas. A mixed finite element method for second order elliptic problems. In I. Galligani and E. Magenes, editors, Mathematical Aspects of the Finite Element Method, pages 292-315, Berlin-Heilderberg-New York, 1977. Springer-Verlag.

[18] K. Stüben. Algebraic multigrid (AMG): experiences and comparisons. Appl. Math. Comput., 13:419-452, 1983. 